\title{
Inhaled IFN- $\gamma$ for persistent nontuberculous mycobacterial pulmonary disease due to functional IFN- $\gamma$ deficiency
}

\author{
T.S. Hallstrand*, H.D. Ochs*, Q. Zhu*, W.C. Liles*
}

Inhaled IFN- $\gamma$ for persistent nontuberculous mycobacterial pulmonary disease due to functional IFN- $\gamma$ deficiency. T.S. Hallstrand, H.D. Ochs, Q. Zhu, W.C. Liles. (C)ERS Journals Ltd 2004.

ABSTRACT: Pulmonary infection with nontuberculous mycobacteria (NTM) in previously healthy human immunodeficiency virus-seronegative individuals is difficult to treat. Recently, functional interferon (IFN)- $\gamma$ deficiency has been identified in individuals susceptible to this disease. Treatment with inhaled IFN- $\gamma$ for NTM pulmonary disease associated with functional IFN- $\gamma$ deficiency has not been previously described.

In this study, the IFN- $\gamma$ pathway was characterised in an individual who had progressive NTM pulmonary infection, despite appropriate multidrug antibiotic therapy, and 10 healthy controls. Levels of IFN- $\gamma$ and tumour necrosis factor- $\alpha$ in whole blood were assessed before and after incubation with lipopolysaccharide, heatkilled Escherichia coli, heat-killed Staphylococcus aureus and phorbol myristate acetate/ionomycin. The coding regions of interleukin (IL)-12, IL-18 and the IL-12 receptor were sequenced using nested primers. IFN- $\gamma 1 \mathrm{~b}\left(100 \mu \mathrm{g} \cdot \mathrm{dose}^{-1}\right)$ was administered to the affected individual by ultrasonic nebuliser 3 days $^{-w^{\prime}} \mathrm{eek}^{-1}$ for 3 months.

In vitro whole blood production of IFN- $\gamma$ with and without physiological stimuli was consistent with functional IFN- $\gamma$ deficiency in the affected individual. There was no evidence of mutation in the coding regions of IL-12p35, IL-12p40, IL-12Rß1 and IL-18 in the affected individual. Treatment with inhaled IFN- $\gamma$ resulted in rapid and sustained clearance of the organism from the airways and stabilisation of lung function.

In conclusion, inhaled interferon- $\gamma$ can be effective for the treatment of nontuberculous mycobacteria pulmonary disease associated with functional interferon- $\gamma$ deficiency.

Eur Respir J 2004; 24: 367-370.
Depts of *Medicine and ${ }^{\#}$ Paediatrics, University of Washington, Seattle, WA, USA.

Correspondence: T.S. Hallstrand

Division of Pulmonary and Critical Care

Dept of Medicine

University of Washington

Box 356522

1959 NE Pacific Street

Seattle

WA 98195

USA

Fax: 12066858673

E-mail: tealh@u.washington.edu

Keywords: Bronchiectasis

host defences

interferon- $\gamma$

mycobacterium

treatment

tumour necrosis factor- $\alpha$

Received: March 292004

Accepted: April 272004

This study was supported by the National Institutes of Health grants HL04231 (T.S. Hallstrand) and HL62995 (W.C. Liles).
Progressive pulmonary disease caused by infection with nontuberculous mycobacteria (NTM) in human immunodeficiency virus (HIV)-seronegative patients without preexisting structural lung disease is a well-described entity [1]. Infection with NTM, usually Mycobacterium avium complex, occurs predominantly in the right middle lobe and lingual of nonsmoking, middle-aged Caucasian females [1]. Bronchiectasis and obstructive lung disease occur as sequelae of persistent infection. The occurrence of this disorder in a well-defined population suggests an acquired or genetic deficiency in host immunity; however, the underlying molecular mechanism has not been fully delineated [2].

Interferon (IFN)- $\gamma$ plays a key role in host defence against mycobacterial disease via macrophage activation [3]. Complete absence of the IFN- $\gamma$ receptor impairs the ability to form mature granulomas and leads to severe disseminated mycobacterial infections in infancy [4, 5]. Low in vitro production of IFN- $\gamma$ has been described in HIV-seronegative patients with NTM pulmonary disease $[6,7]$. The cause of inadequate IFN- $\gamma$ production in this patient population is unknown. Deficiency of the interleukin (IL)-12 receptor leads to decreased IFN- $\gamma$ production by natural killer (NK) and Tcells, and mutation of this receptor has been described in systemic mycobacterial disease, with preservation of the ability to form granulomas [8]. Production of IFN- $\gamma$ is also dependent on IL-18, and deficiency of IL-18 in a murine model leads to reduced granuloma formation in response to mycobacterial infection [9]. Recently, two patients were described with NTM pulmonary infection and abnormally low in vitro IFN- $\gamma$ production, but normal-to-high IFN- $\gamma$ staining in $\mathrm{CD} 3+\mathrm{T}$-cells, implicating defective IFN- $\gamma$ secretion [10]. Since the IFN- $\gamma$ receptor is unaffected in these instances in which IFN- $\gamma$ production is reduced, NTM pulmonary disease may be amenable to IFN- $\gamma$ replacement therapy.

In this report, the authors describe an individual who presented in late adulthood with progressive bronchiectasis and recurrent pulmonary infections caused by NTM, initially $M$. avium complex, followed by $M$. abscessus. Despite aggressive, appropriate multidrug antimicrobial treatment, the patient deteriorated clinically and continued to have high concentrations of mycobacteria in her sputum. In vitro production of IFN- $\gamma$ at baseline and in response to endotoxin and bacterial antigens was reduced. Treatment of this individual with inhaled IFN- $\gamma$ resulted in clinical stabilisation and clearance of mycobacteria from sputum. 


\section{Case presentation}

A 51-yr-old Caucasian female developed cough, sputum production and low-grade fevers. Six years previously, she had been treated for $M$. avium complex infection with ciprofloxacin, rifampin and ethambutol for 12 months. Prior to $M$. avium complex infection, she had no history of chronic lung disease or recurrent infections. She had a remote 10 pack-yr smoking history. A chest radiograph revealed multiple nodules throughout both lung fields, and consolidation of the left lower lobe and lingula. A computed tomography scan demonstrated peribronchial or "tree-inbud" pattern infiltrates and widespread cylindrical bronchiectasis. A sputum sample revealed 3+ mucoid Pseudomonas aeruginosa and 4+ M. abscessus. After successful treatment of $P$. aeruginosa with tobramycin and ceftazidime, treatment for M. abscessus was initiated with cefoxitin, clarithromycin and amikacin, based on drug-sensitivity testing. Despite various three-drug treatment regimens, she continued to have high concentrations of $M$. abscessus in her sputum over 19 months of treatment and developed worsening obstructive lung disease.

Laboratory evaluation for immunodeficiency was negative, including normal serum values for total immunoglobulin (Ig)G and IgG subclasses, total IgE, CH50, C3 and C4, and normal neutrophil and lymphocyte counts in peripheral blood. Sweat chloride was $8 \mathrm{mEq} \cdot \mathrm{L}^{-1}$ (normal $<60 \mathrm{mEq} \cdot \mathrm{L}^{-1}$ ). Genetic analysis revealed that the patient was a carrier of the $\Delta \mathrm{F} 508$ mutation of the cystic fibrosis transmembrane conductance regulator gene. Serological testing for HIV was negative. The neutrophil oxidative burst was normal, as was T-cell proliferation to tetanus, candida and phytohemagglutinin. Antibody titres to rubella and rubeola were normal, and intradermal skin testing showed anergy to candida, mumps and trichophyton. Additional laboratory investigations were conducted to characterise the IFN- $\gamma$ pathway.

\section{Methods}

\section{In vitro whole blood cytokine assays}

Venous blood samples were obtained from the affected individual and 10 healthy volunteers (five males; five females; age range 28-58 yrs). Each participant gave written informed consent and the University of Washington Human Subjects Review Committee approved the study procedures. Heparinised venous blood was diluted 1:5 in RPMI 1640 (GIBCOInvitrogen Corp., Grand Island, NY, USA) and incubated for $18 \mathrm{~h}$ at $37^{\circ} \mathrm{C}$ in a $5 \% \mathrm{CO}_{2}$-humidified cell culture incubator with lipopolysaccharide (LPS; $100 \mathrm{ng} \cdot \mathrm{mL}^{-1}$; from $E$. coli O111:B4 (Sigma, St Louis, MO, USA)), heat-killed E. coli $\left(5 \times 10^{6} \mathrm{cfu} \cdot \mathrm{mL}^{-1}\right)$, heat-killed $S$. aureus $\left(5 \times 10^{6} \mathrm{cfu} \cdot \mathrm{mL}^{-1}\right)$, phorbol myristate acetate (PMA; $10 \mathrm{ng} \cdot \mathrm{mL}^{-1}$; Sigma) and ionomycin $\left(500 \mathrm{ng} \cdot \mathrm{mL}^{-1}\right.$; Sigma). After incubation, culture supernatants were removed for determination of IFN- $\gamma$ and tumour necrosis factor (TNF)- $\alpha$ concentrations by cytokinespecific ELISA (DuoSet paired antibody reagents; R\&D Systems, Minneapolis, MN, USA).

\section{Sequence analysis of $I L-12, I L-12 \beta 1$ and $I L-18$}

Total RNA was isolated from peripheral blood mononuclear cells with Trizol (Invitrogen, Carlsbad, CA, USA). The first strand cDNA was synthesised using the Omniscript Kit (Quiagen, Valencia, CA, USA) according to the manufacturer's instructions. The cDNAs of IL-12p35, IL$12 \mathrm{p} 40$, IL-12R $\beta 1$ and IL-18 were amplified by polymerase chain reaction (PCR) using the following primers: IL-12p35, 5'-AAAGCAAGAGACCAGGTCC (forward) and 5'-CCC TCCCTAGTTCTTAATCC (reverse); IL-12p40, 5'-GTTT CAGGGCCATTGGACTC (forward) and 5'-TGGCAAC TTGAGAGCTGGAA (reverse); IL-12R $\beta 1$, 5'-TGAACC TCGCAGGTGGCAGA (forward) and 5'-AGCCTCGGGC GAGTCACTCA (reverse); and IL-18, 5'-GCCTGGA CAGTCAGCAAGGA (forward) and 5'-CCAGGCTAG AGCGCAATGGT (reverse). Purified PCR products were sequenced with the BigDye Terminator Cycle Sequencing Kit (Perkin-Elmer, Foster City, CA, USA) using sets of nested primers (available on request).

\section{IFN- $\gamma$ administration}

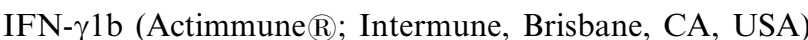
was administered 3 days $\cdot$ week $^{-1}$ at a dose of $100 \mu \mathrm{g}(0.5 \mathrm{~mL})$ diluted in $4 \mathrm{~mL}$ of sterile water by ultrasonic nebuliser (Pari LC Plus, Monterey, CA, USA). Spirometry was performed before and $15 \mathrm{~min}$ after the initial dose.

\section{Results}

In vitro whole blood production of IFN- $\gamma$ and $\mathrm{TNF}-\alpha$ from the affected individual was compared to that of 10 healthy control volunteers (table 1). Physiological stimuli, including LPS, heat-killed E. coli and heat-killed $S$. aureus, induced production of both IFN- $\gamma$ and TNF- $\alpha$ in blood from normal volunteers. In contrast, physiological stimuli failed to induce production of detectable IFN- $\gamma$ in blood from the affected

Table 1. - In vitro production of interferon (IFN)- $\gamma$ and tumour necrosis factor (TNF)- $\alpha$ from the affected individual and 10 healthy control volunteers

$\begin{array}{ccccc}\text { Control }^{\#} & \text { LPS } & \begin{array}{c}\text { E. coli } \\ 5 \times 10^{6} \cdot \mathrm{mL}^{-1}\end{array} & \begin{array}{c}\text { S. aureus } \\ 5 \times 10^{6} \cdot \mathrm{mL}^{-1}\end{array} & \begin{array}{c}\text { PMA } 10 \mathrm{ng} \cdot \mathrm{mL}^{-1}+ \\ \text { ionomycin } 500 \mathrm{ng} \cdot \mathrm{mL}^{-1}\end{array}\end{array}$

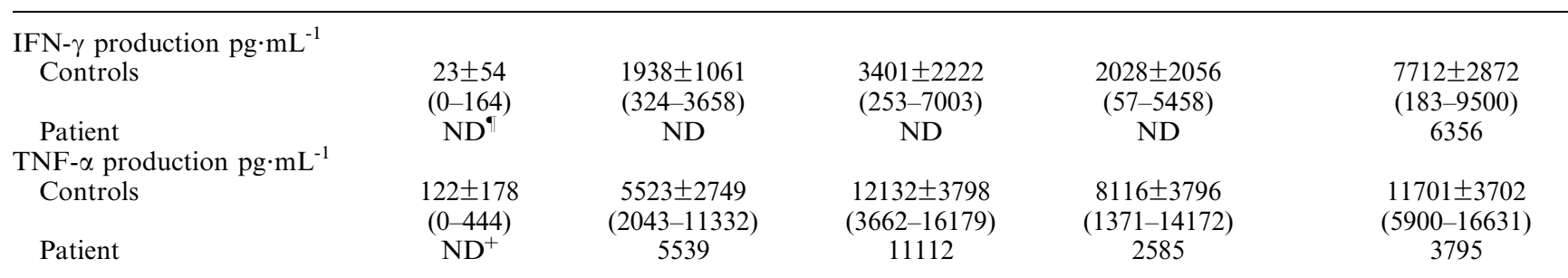

Data are presented as mean \pm SD (range) and n. LPS: lipopolysaccharide; E. coli: Escherichia coli; S. aureus: Staphylococcus aureus; PMA: phorbol myristate acetate; ND: none detected. \#: no stimulus; $\stackrel{\uparrow}{:}<15 \mathrm{pg} \cdot \mathrm{mL}^{-1} ;{ }^{+}:<8 \mathrm{pg} \cdot \mathrm{mL}^{-1}$. 


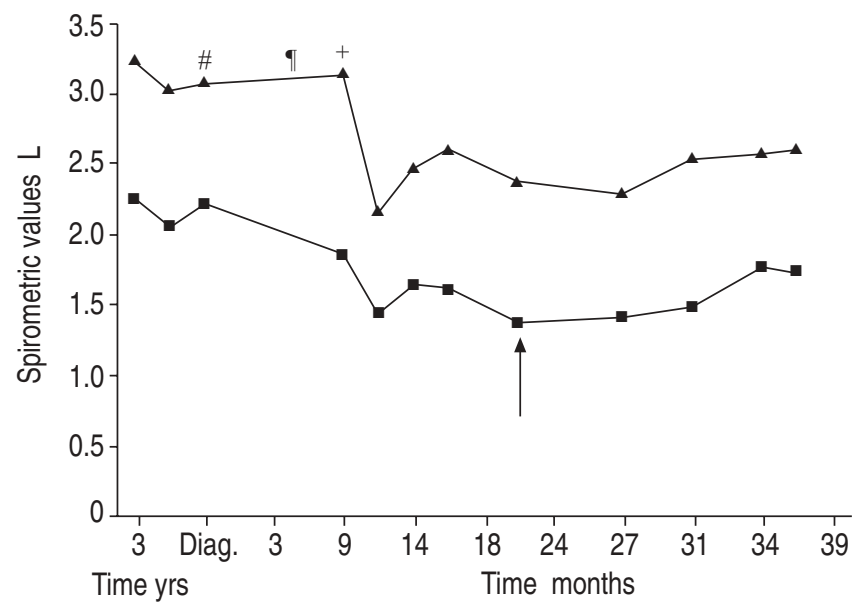

Fig. 1.-Lung function results (forced expiratory volume in one second (ם) and forced vital capacity $(\boldsymbol{\Delta})$ ) during infection with Mycobacterium abscessus, before and after the initiation of interferon$\gamma$ therapy (arrow) for 3 months. Diag.: diagnosis. \#: cefoxitin, clarithromycin and i.v. amikacin; : imipenem, clarithromycin and inhaled amikacin; ${ }^{+}$: levofloxacin, clarithromycin and inhaled amikacin.

individual; inducible expression of TNF- $\alpha$ was similar to that of normal volunteers. Production of IFN- $\gamma$ and TNF- $\alpha$ in response to nonspecific stimulation with PMA/ionomycin was comparable between the affected individual and normal volunteers. Sequence analysis of the coding regions of IL$12 \mathrm{p} 35$, IL-12p40, IL-12R $\beta 1$ and IL-18 revealed no evidence of mutation in the affected individual.

Prior to initiation of inhaled IFN- $\gamma$, sputum cultures were persistently positive for $M$. abscessus, despite appropriate

Table 2.-Sputum culture results during infection with Mycobacterium abscessus, before and after the initiation of interferon- $\gamma$ therapy at 22 months for a duration of 3 months

Time after diagnosis

months

\begin{tabular}{lcc}
\cline { 2 - 3 } months & Stain & Culture \\
\hline 0 & $4+$ & $2+$ \\
$0.5^{\bullet}$ & $2+$ & $2+$ \\
7 & $2+$ & $2+$ \\
9 & $4+$ & $2+$ \\
12 & $4+$ & $3+$ \\
14 & $1+$ & $2+$ \\
16 & $3+$ & $2+$ \\
18 & $3+$ & $2+$ \\
24 & neg & $5^{\#}$ \\
27 & neg & $2^{\#}$ \\
29 & neg & neg \\
31 & neg & neg \\
33 & neg & neg \\
34 & neg & neg \\
37 & neg & neg \\
39 & neg & neg \\
\hline
\end{tabular}

Acid-fast bacilli (AFB) stains were classified according to the following scale: $1+\left(>10\right.$ AFB $\cdot$ smear $^{-1}$, but $<1$ AFB $\cdot 200 \times$ field $\left.^{-1}\right) ; 2+(1-9$ AFB $200 \times$ field $\left.^{-1}\right) ; 3+\left(>10\right.$ AFB $200 \times$ field $^{-1}$, but $<10$ AFB $500 \times$ field $\left.^{-1}\right) ; 4+\left(>10\right.$ AFB $\cdot 500 \times$ field $\left.^{-1}\right)$. AFB cultures were classified according to the following scale: $1+\left(50-100\right.$ colonies $^{-1}$ plate $\left.{ }^{-1}\right) ; 2+$ (100-200 colonies $\cdot$ plate $\left.^{-1}\right) ; 3+\left(200-500\right.$ colonies plate $\left.^{-1}\right) ; 4+(>500$ colonies plate ${ }^{-1}$ ). neg: negative, i.e. no AFB detected on the stain or no colonies identified in the culture. ${ }^{\#}$ : number of colonies on plate; ${ }^{\circ}: 0.5$ months corresponds to 2 weeks. multidrug antimycobacterial treatment, and lung function progressively deteriorated over a period of 20 months (fig. 1; table 2). Following initiation of inhaled IFN- $\gamma$ therapy, there was rapid clearance of the organism from the airways and stabilisation of lung function. After 3 months of inhaled IFN$\gamma$, antimycobacterial treatment was continued for an additional 9 months.

\section{Discussion}

A well-defined group of patients, predominantly middleaged females without pre-existing structural lung disease or immunodeficiency, may account for the majority of pulmonary infections caused by NTM [2, 11]. Recent evidence shows that reduced production of IFN- $\gamma$ by T- and NK cells may predispose these patients to mycobacterial infection $[6$, 7]. In this report, it is shown that pulmonary NTM infection associated with impaired in vitro IFN- $\gamma$ production can be successfully treated with inhaled IFN- $\gamma$.

Susceptibility to NTM pulmonary disease in a well-defined group of patients suggests an innate or acquired defect in host defence. Major defects in the IFN- $\gamma$ pathway, including abnormalities of the receptors for IFN- $\gamma$ and IL-12, have been associated with disseminated infection caused by weakly pathogenic mycobacteria, such as bacille Calmette-Guerin and NTM $[4,5,8]$. Neither of two known mutations of the IFN- $\gamma$ receptor 1 was identified in a study of patients with NTM pulmonary disease [12]. Evidence of low IFN- $\gamma$ production, in response to endotoxin, antigen and mitogen in patients with NTM pulmonary disease, suggests that the defect in host defence is in the pathway leading to IFN- $\gamma$ production and secretion [6, 7]. The current authors characterised the receptors for IL-12 and IL-18, which play a major role in the activation of immune cells to produce IFN- $\gamma$, and found normal sequences of these receptors, despite nondetectable in vitro levels of IFN- $\gamma$ at baseline, and following stimulation with endotoxin and bacterial antigens. These results are consistent with the observation that, despite low extracellular levels of IFN- $\gamma$, intracellular levels of IFN- $\gamma$ in T-cells were normal to high, suggesting an abnormality in the transport or secretion mechanism for IFN$\gamma[10]$.

Treatment of NTM pulmonary disease with multidrug antimycobacterial therapy frequently fails. In one case study, negative sputum cultures were achieved in only seven out of 24 patients over a 1-yr follow-up period, as a result of either drug intolerance or development of drug resistance [2]. Evidence of low IFN- $\gamma$ production in this disease process suggests a rational role for IFN- $\gamma$ replacement therapy. IFN- $\gamma$ has been approved by the US Food and Drug Administration for treatment of chronic granulomatous disease and malignant osteopetrosis. Previous reports have shown that subcutaneous IFN- $\gamma$ was effective in reducing or eliminating disseminated $M$. avium complex infections in both HIVseronegative [13] and HIV-infected patients [14]. Treatment of multidrug-resistant tuberculosis with aerosolised IFN- $\gamma$ $\left(500 \mu \mathrm{g} 3\right.$ times $^{-}$week $^{-1}$ ) temporarily reduced mycobacterial burden [15]. Aerosolised IFN- $\gamma\left(500 \mu \mathrm{g} 3\right.$ times $\cdot$ week $\left.^{-1}\right)$ reduced the burden of $M$. avium in a patient with pre-existing cavitary lung disease from advanced silicosis [16].

This is the first study to report the use of inhaled interferon$\gamma$ for persistent nontuberculous mycobacteria pulmonary disease in an individual with low in vitro production of interferon- $\gamma$. Treatment with inhaled interferon- $\gamma$ was welltolerated, and resulted in microbiological cure and clinical resolution of disease. This finding suggests that inhaled interferon- $\gamma$ therapy should be considered in individuals 
with nontuberculous mycobacteria infection that progresses despite conventional treatment. Additionally, when appropriate resources are available, laboratory evaluation should be performed to identify functional interferon- $\gamma$ deficiency, and interferon- $\gamma$ therapy should be considered early in the treatment of affected individuals. Further studies are warranted to determine if functional interferon- $\gamma$ deficiency is a common feature of individuals with progressive nontuberculous mycobacteria disease. The favourable therapeutic results of interferon- $\gamma$ treatment presented in the current report should be confirmed in controlled trials of groups of patients with nontuberculous mycobacteria pulmonary disease, with and without underlying functional interferon- $\gamma$ deficiency.

\section{References}

1. Prince DS, Peterson DD, Steiner RM, et al. Infection with Mycobacterium avium complex in patients without predisposing conditions. $N$ Engl J Med 1989; 321: 863-868.

2. Huang JH, Kao PN, Adi V, Ruoss SJ. Mycobacterium avium-intracellulare pulmonary infection in HIV-negative patients without preexisting lung disease: diagnostic and management limitations. Chest 1999; 115: 1033-1040.

3. Chan J, Xing Y, Magliozzo RS, Bloom BR. Killing of virulent Mycobacterium tuberculosis by reactive nitrogen intermediates produced by activated murine macrophages. J Exp Med 1992; 175: 1111-1122.

4. Dorman SE, Holland SM. Mutation in the signal-transducing chain of the interferon-gamma receptor and susceptibility to mycobacterial infection. J Clin Invest 1998; 101: 2364-2369.

5. Jouanguy E, Altare $\mathrm{F}$, Lamhamedi $\mathrm{S}$, et al. Interferongamma-receptor deficiency in an infant with fatal bacille Calmette-Guerin infection. N Engl J Med 1996; 335: 19561961.

6. Safdar A, White DA, Stover D, Armstrong D, Murray HW. Profound interferon gamma deficiency in patients with chronic pulmonary nontuberculous mycobacteriosis. Am $J$ Med 2002; 113: 756-759.

7. Greinert U, Schlaak M, Rusch-Gerdes S, Flad HD, Ernst M. Low in vitro production of interferon-gamma and tumor necrosis factor-alpha in HIV-seronegative patients with pulmonary disease caused by nontuberculous mycobacteria. J Clin Immunol 2000; 20: 445-452.

8. Altare F, Durandy A, Lammas D, et al. Impairment of mycobacterial immunity in human interleukin-12 receptor deficiency. Science 1998; 280: 1432-1435.

9. Sugawara I, Yamada H, Kaneko H, Mizuno S, Takeda K, Akira S. Role of interleukin-18 (IL-18) in mycobacterial infection in IL-18-gene-disrupted mice. Infect Immun 1999; 67: 2585-2589.

10. Safdar A, Armstrong D, Murray HW. A novel defect in interferon-gamma secretion in patients with refractory nontuberculous pulmonary mycobacteriosis. Ann Intern Med 2003; 138: 521.

11. Reich JM, Johnson RE. Mycobacterium avium complex pulmonary disease. Incidence, presentation, and response to therapy in a community setting. Am Rev Respir Dis 1991; 143: $1381-1385$.

12. Huang $\mathrm{JH}$, Oefner PJ, Adi V, et al. Analyses of the NRAMP1 and IFN-gammaR1 genes in women with Mycobacterium avium-intracellulare pulmonary disease. Am J Respir Crit Care Med 1998; 157: 377-381.

13. Holland SM, Eisenstein EM, Kuhns DB, et al. Treatment of refractory disseminated nontuberculous mycobacterial infection with interferon gamma. A preliminary report. $N$ Engl J Med 1994; 330: 1348-1355.

14. Squires KE, Brown ST, Armstrong D, Murphy WF, Murray HW. Interferon-gamma treatment for Mycobacterium aviumintracellular complex bacillemia in patients with AIDS. J Infect Dis 1992; 166: 686-687.

15. Condos R, Rom WN, Schluger NW. Treatment of multidrug-resistant pulmonary tuberculosis with interferongamma via aerosol. Lancet 1997; 349: 1513-1515.

16. Chatte G, Panteix G, Perrin-Fayolle M, Pacheco $Y$. Aerosolized interferon gamma for Mycobacterium aviumcomplex lung disease. Am J Respir Crit Care Med 1995; 152: 1094-1096. 